\title{
Agôn
}

Revue des arts de la scène

Critiques | Saison 2010-2011

\section{Monkey sandwich, chorégraphie de Wim Vandekeybus}

\author{
Lise Lenne
}

\section{(2) OpenEdition}

Journals

Édition électronique

URL : http://journals.openedition.org/agon/1229

DOI : $10.4000 /$ agon. 1229

ISSN : 1961-8581

\section{Éditeur}

Association Agôn

\section{Référence électronique}

Lise Lenne, "Monkey sandwich, chorégraphie de Wim Vandekeybus », Agôn [En ligne], Critiques, mis en ligne le 09 février 2019, consulté le 23 septembre 2020. URL : http://journals.openedition.org/agon/ 1229 ; DOI : https://doi.org/10.4000/agon.1229

Ce document a été généré automatiquement le 23 septembre 2020

Association Agôn et les auteurs des articles 


\title{
Monkey sandwich, chorégraphie de Wim Vandekeybus
}

\author{
Lise Lenne
}

\section{RÉFÉRENCE}

Monkey Sandwich, de Wim Vandekeybus, du 10 au 12 septembre et du 02 au 11 novembre 2010, KVS, Bruxelles

1 Pour son nouveau spectacle, Monkey sandwich, le chorégraphe flamand Wim Vandekeybus nous propose une forme singulière, à la croisée de la danse, du cinéma et de la performance. Mais il procède ici par inversion : alors que nombre de spectacles utilisent de manière ponctuelle l'insertion d'images vidéo, Monkey sandwich commence par la projection, sur l'écran descendu, d'un film réalisé par Wim Vandekeybus luimême, qui dure quasiment tout le temps de la représentation. Ce n'est qu'au bout d'une vingtaine de minutes qu'apparaît sur scène le jeune danseur-performeur Damien Chapelle : dans l'espace-temps de la fiction cinématographique fait soudain irruption le réel d'un corps qui bouge. Le cadre s'ouvre alors pour inclure la réalité vivante de ce corps et de son espace propre : la scène. Le spectacle s'invente, avec la complicité du spectateur, dans la rencontre entre ces deux espaces mis en présence, dans le dialogue à fleur de peau du corps et de l'image.

Monkey sandwich, chorégraphie de Wim Vandekeybus 


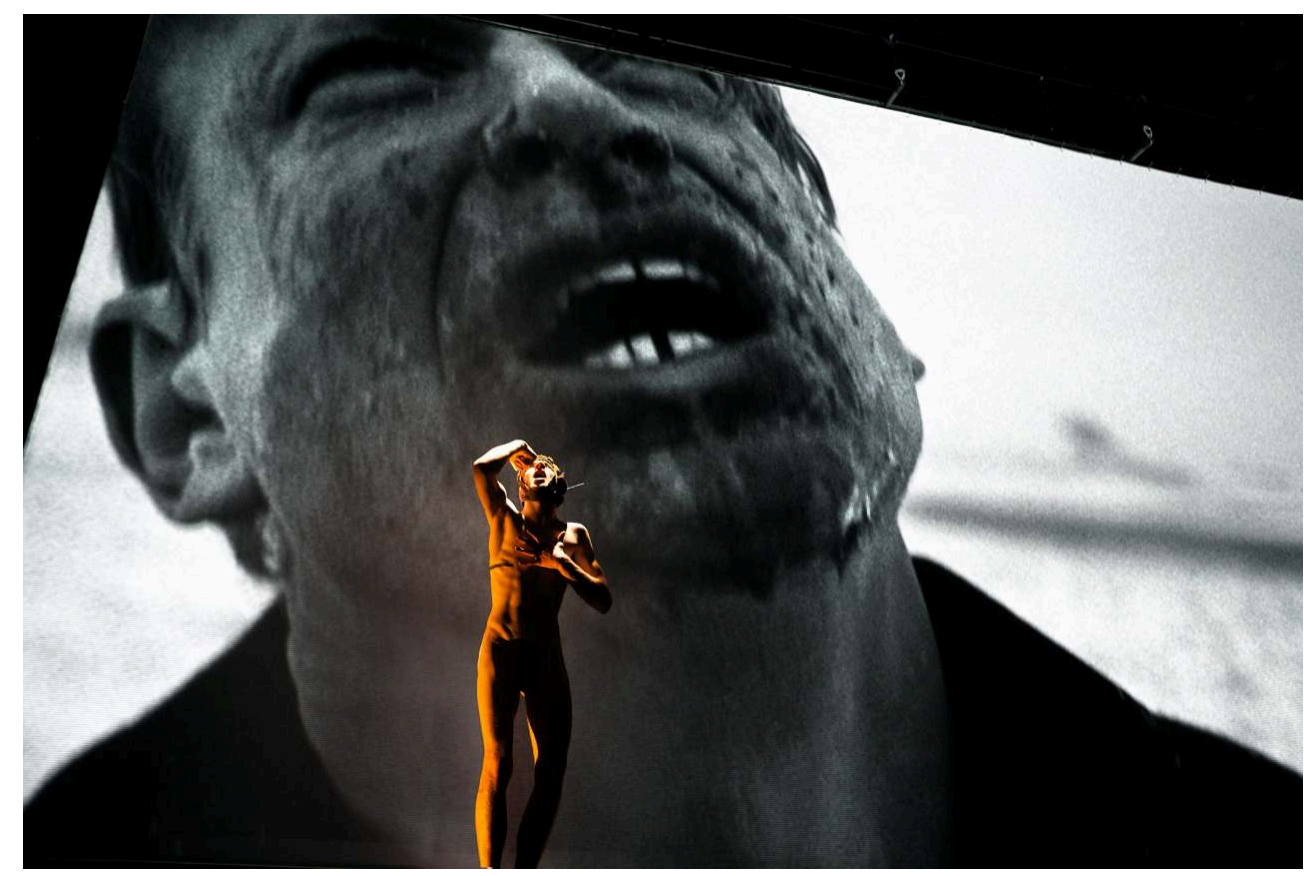

(C) DANNY WILLEMS

2 «Monkey sandwich », c'est l'équivalent de l'expression flamande "broodje aap », ou légendes urbaines en français. Le spectacle questionne notre rapport aux histoires : des mythes fondateurs, qui se jouent et se rejouent encore, au creux de nos fantasmes les plus réels, à la petite anecdote, comme celle du doigt coupé, que l'on raconte - qui nous raconte - et qui, à chaque fois, prend une couleur différente selon l'oreille à qui elle est destinée. Le film met ainsi bout à bout quatre fragments que Wim Vandekeybus nous laisse libres de raccorder ou non: en effet, chaque passage nous montre les errances d'un personnage, incarné à chaque fois avec force par le même acteur, l'anglais Jerry Killick, mais le film ne nous dit jamais si l'on suit une ou plusieurs histoires, un ou plusieurs personnages. Traversé par trois mouvements de bascule d'un ordre de réalité à un autre, il joue donc de la variante et du miroir déformant pour aborder à chaque fois, sous un angle décalé, le même sujet. Dans la première partie, Jerry Killick apparaît sous les traits d'un metteur en scène autoritaire, voire tyrannique, qui croit pouvoir nier le jeu, l'écart qui oppose, comme deux faces d'un même miroir, l'art et la réalité. Dans une scène d'une cruauté désarmante, il exige d'un de ses acteurs qu'il «bouffe » sa partenaire, jusqu'à ce qu'il ne fasse plus semblant, jusqu'à ce qu'il ne joue plus. À travers cette scène, filtre la question souterraine que Vandekeybus se (et nous) pose : où se situe le point de bascule, et donc le point de rencontre, entre fiction et réalité, entre mon désir et sa réalisation, entre moi et l'autre ? À quel moment est-ce que je deviens responsable? Coupable? En effet, la deuxième partie du film s'ouvre sur une reconversion, ou plutôt une renaissance : la figure du metteur en scène disparait pour laisser place à une autre figure, celle du maittre d'œuvre, d'un homme qui un beau jour décide de fonder d'un même geste une famille et un village. Est-ce le même homme dont la vie est soudain traversée par une rupture radicale ? Quelle est la nature du lien qui unit ces deux histoires? Le film nous laisse l'imaginer et l'interpréter. Et l'on glisse alors peu à peu dans un univers aux accents mythologiques et un onirisme affirmé. On y voit l'homme, tel Prométhée, qui tente de faire surgir de la terre et des eaux, une utopie : au sein de ces communautés (le village, la famille), ce sont les histoires que l'on 
se raconte qui font office de ciment. Mais ce ciment-là est humain - trop humain dira-ton - et le geste de l'homme trop grand, démesuré, insensé. Le créateur s'absente et la nature finit par reprendre ses droits: les digues, construites autour du village pour retenir les eaux, cèdent sous le déluge, qui engloutit les habitants avec leur histoires et leurs rivalités. Scène de chute qui ouvre sur une troisième partie, onirique, centrée sur la culpabilité du protagoniste, prisonnier de ses cauchemars et hanté par la mort de son enfant qu'il cherche à retrouver. Lorsqu'il croit la revoir, ce ne sont que des coups qui lui sont rendus, le laissant à sa solitude et à ses fantômes. Sur scène, dans un espacetemps qui semble se situer en-deçà ou au-delà du langage, c'est cette même solitude qui nous est donnée à voir à travers la performance solitaire de Damien Chapelle, vivant écho de ce qui se joue sur l'écran. Solitude animale (qui entre en résonance avec la scène de chasse à l'homme qui clôture le film), traversée par le désir tenace de reconstruire, recoller les éclats brisés d'un monde enfui, d'entrer en dialogue avec cet autre inatteignable: celui de l'écran, celui du public, celui à l'intérieur de soi. En témoignent ses apostrophes répétées à son double filmé, ses bouts de texte, de mots, de sons découpés dans plusieurs langues, ces êtres de papier qu'il fabrique avec frénésie pour peupler une scène trop vide, mais aussi ses plongées à répétition dans un aquarium, comme pour retourner à l'état foetal : hors de toute atteinte, hors du monde. Un monde que l'on ne parvient plus à habiter par manque de réalité. À la fin, le géant de l'écran, projeté dans le réel de la scène, ne fait pas long feu et finit par disparaître, avalé par le plateau, laissant l'autre à son inaltérable solitude et les spectateurs devant l'image troublante d'un aquarium qui se vide, des larmes d'un tuyau d'arrosage et du cri d'un homme, devant le noir qui soudain se fait.

3 La proposition que nous fait ici Wim Vandekeybus, avec force et subtilité, est celle d'un voyage qui nous met face à notre humanité - donc face à nos désirs toujours trop grands pour le monde qui nous contient. On peut se sentir par moments perdu dans la matière dense et hétéroclite d'une forme singulière qui interpelle, interroge, nous fait douter; mais aussi captivé, pris d'assaut par certains passages, filmés ou incarnés, d'une cruauté innommable, d'une beauté crue, sans fard. Monkey sandwich montre sans désigner et - c'est là son tour de force - nous laisse la liberté de le traverser, de le voir, de l'entendre. Il nous fait interprètes. Nous donne, à nous spectateurs, une place pour exister et participer à l'élaboration d'un sens. Un spectacle conçu comme un espace que l'on pourrait - enfin - habiter.

4 Tournée : le 21 janvier 2011, Maison de la Culture d'Amiens 